\title{
Co-Inocula Assays of Yeasts with "Killer" Pheno-Type and Sensitive Strains of Saccharomyces cere-visiae with Defects in Mannoprotein Synthesis ${ }^{\dagger}$
}

\author{
Patricia Gil 1,*, Alberto Martínez ${ }^{1}$, Joaquín Bautista ${ }^{1}$, Rocío Velázquez ${ }^{2}$, Manuel Ramírez ${ }^{1}$ and \\ Luis Miguel Hernández ${ }^{1}$
}

Citation: Gil, P.; Martínez, A.; Bautista, J.; Velázquez, R.; Ramírez, M.; Hernández, L.M. Co-Inocula Essays of Yeasts with "Killer" Phenotype and Sensitive Strains of Saccharomyces cerevisiae with Defects in Mannoprotein Synthesis. 2021, 70, 38. https://doi.org/10.3390/foods_202007732

Published: 10 November 2020

Publisher's Note: MDPI stays neutral with regard to jurisdictional claims in published maps and institutional affiliations.

Copyright: (c) 2020 by the authors. Licensee MDPI, Basel, Switzerland. This article is an open access article distributed under the terms and conditions of the Creative Commons Attribution (CC BY) license (http://creativecommons.org/licenses/by/4.0/)
1 Departamento de Ciencias Biomédicas, Área de Microbiología, Facultad de Ciencias, Universidad de Extremadura, 06006 Badajoz, Spain; albertomb@unex.es (A.M.); joaquinbg@unex.es (J.B.); mramirez@unex.es (M.R.); lmhernan@unex.es (L.M.H.)

2 Dipartimento Scienze Biomediche, Università degli Studi di Padova, 35020 Padua, Italy; rociovelazquez1981@gmail.com

* Correspondence: patriciagf@unex.es

† Presented at the 1st International Electronic Conference on Food Science and Functional Foods, 10-25 November 2020; Available online: https://foods_2020.sciforum.net/.

\begin{abstract}
Yeast mannoproteins have been thoroughly studied in recent years due to their contribution to different properties of wines. Our working group has the aim to establish the possible relationship between the structure of the mannoprotein (size and charge) and its effect on wines. For this, we have different non-transgenic mutants that synthesize mannoproteins with altered but known structures (mnn mutants). We have constructed double mutants with each of the available single mutants, thus obtaining a very diverse collection of mannoproteins that differ in size and charge. Since the mutants are unable to complete wine fermentations satisfactorily, an alternative is to grow mutants in the laboratory, purify their mannoproteins and add them later to wines. Another option would be to use co-inocula of the mentioned mutants, together with "killer" wine yeast strains capable of killing the mutants. In this work, experiments were carried out to determine the behavior of different "killer" wine strains with each one of the defective mutants in mannoprotein synthesis. The yeast Torulaspora delbrueckii Killer (Kbarr1) has been proved to be especially suitable for making this type of co-inoculum.
\end{abstract}

Keywords: mannoprotein; Saccharomyces cerevisiae; mnn mutants; killer phenotype

\section{Introduction}

In recent years, the effect of mannoproteins on wine has been extensively studied mainly due to the high demand for wines aged on lees. This method is mainly used in white barrel-fermented and natural sparkling wine, among others. This ecological process aims to improve several characteristics of the wine: the quality of the foam, the mouthfeel, the aroma, protein stability and other related features, through the chemical interactions between mannoproteins and wine compounds. The yeasts release some mannoproteins during the fermentation, but mainly during aging, when yeasts undergo autophagy followed by cell death and subsequent autolysis [1,2].

In our laboratory, we are trying to establish a relationship between the charge size of the mannoproteins and its effects on some aspects of wine. For that purpose, we have used mannan-defective single mutants ( $\mathrm{mnn}$ ) [3] to make double mutants with every possible combination of single mutations, thus, we can obtain may different mannoprotein structures. However, these strains are not adequate to perform complete wine fermentation, so the mannoproteins must be purified from laboratory cultures and added to the wines. 
Another way to get mannoproteins from the mutants released into the wine is to design fermentation strategies using co-inocula of a killer strain [4] with high fermentative efficiency and a sensitive mnn mutant. Killer strains are very common in yeast used in enology, so they guarantee the completion of fermentation and they kill mnn mutants, thus accelerating the release of mannoproteins. In our laboratory, we have a line of research focused on killer strains $[5,6]$ which allows us to have a wide collection of killer yeasts.

The aim of this work is to check the effect of every killer strain on every one of the mannan-defective mutants under different conditions. The results of these experiments will be very useful to decide the best combinations of strains to be used in the main fermentation of wine or second fermentation in CAVA, Spanish sparkling wine elaborate by Champenoise method.

\section{Materials and Methods}

Saccharomyces cerevisiae X2180 and single mutants with defects in the structure of mannoproteins $m n n 1, m n n 2, m n n 3, m n n 5, m n n 6, m n n 9$ and $m n n 10$ [3] are from the laboratory collection (Figure 1). Double mutants: mnn1mnn2, mnn1mnn6, mnn1mnn9, mnn $1 m n n 10, m n n 2 m n n 6, m n n 2 m n n 10$ and mnn6mnn10. They are obtained by the conjugation of haploid strains and then the selection of spores.

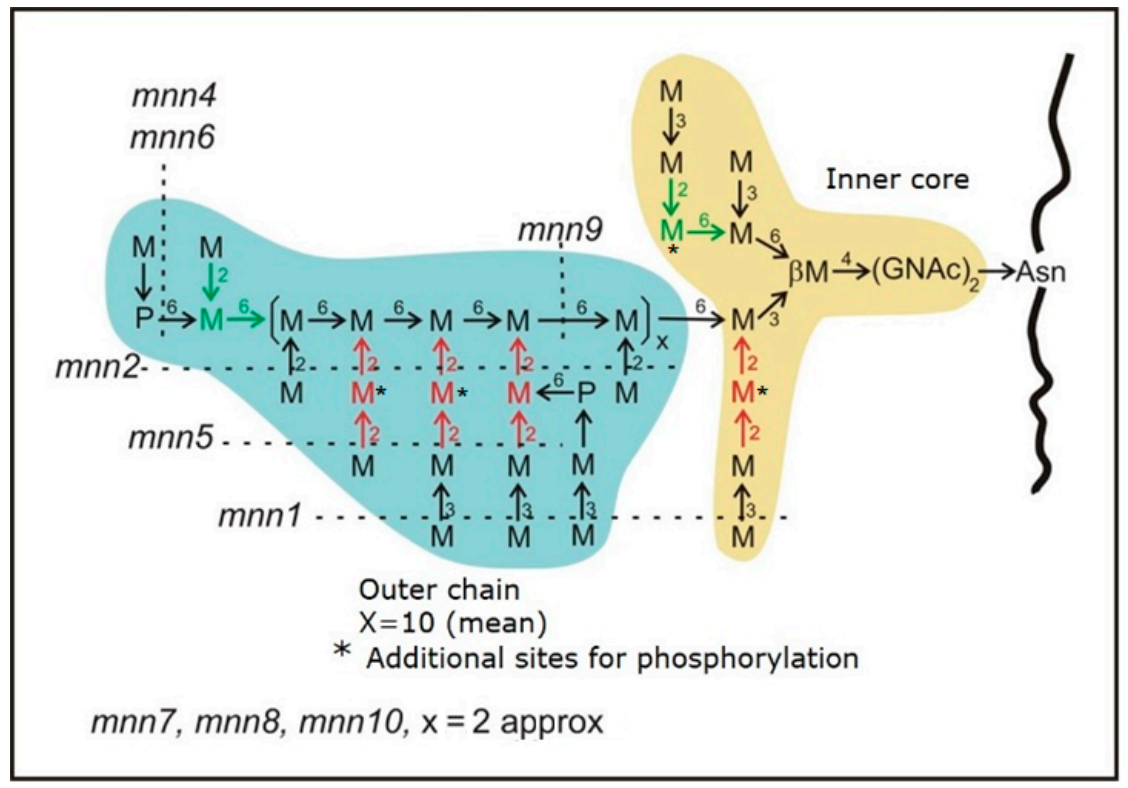

Figure 1. Chemotypes of mannoprotein mutants [3].

Saccharomyces cerevisiae (S.c) killer strains: F166 (K1), E7AR1 (K2), F182 (K28), EX229 (Klus). Torulaspora delbruekii (T.d) killer strains: EX1180 (Kbarr1), EX1257 (Kbarr2). As nokiller control: EX1180-2K- $\left(\right.$ T.d, $\left.\mathrm{K}^{\mathrm{S}}\right)$ and EX33 (S.c, $\left.\mathrm{K}^{\mathrm{S}}\right)[5,6]$.

All yeasts were grown in YEPD (1\% yeast extract, $2 \%$ peptone, $2 \%$ dextrose) liquid medium at room temperature with agitation until the late exponential phase of growth. Then, the mutant yeasts were spread in YEPD-methylene blue plates and killer yeasts were dropped on top. The plates were checked every day and photographs were taken during the process.

The killer effect of the yeasts in the patches was determined by observing the halos generated by the death of the mutants (Figure 2). 


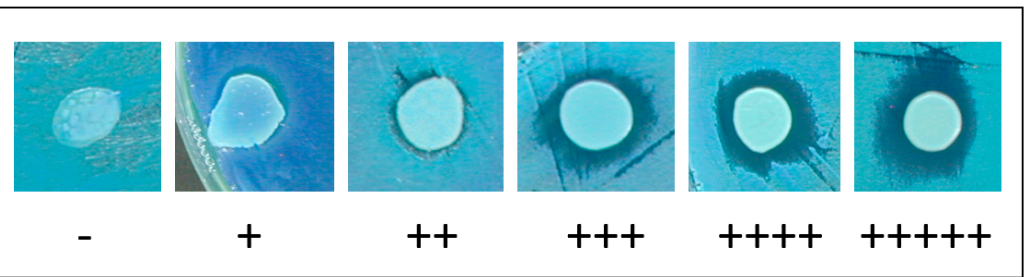

Figure 2. Punctuations according to the halo produced by the death of the mutants due to the toxin.

\section{Results and Discussion}

\subsection{The More Affected the Mannoprotein Is, the More Sensitive It Is to the Killer Toxin}

In light of the results, it seems clear that, independently of the conditions, the sensitivity to the toxin is higher when the mutation leads to a reduction in size of N-linked chains (Table 1), probably because it facilitates the entry of the toxin or the interaction with its receptor.

Table 1. Kbarr1 killer effect at different $\mathrm{pH}$ and temperatures.

\begin{tabular}{ccccccccccc}
\hline pH & $\mathrm{T}$ & wt & mnn1 & mnn2 & mnn3 & mnn5 & mnn6 & mnn9 & mnn10 \\
\hline \multirow{2}{*}{3.5} & $13^{\circ} \mathrm{C}$ & ++ & + & ++ & +++ & ++ & ++ & +++++ & ++++ \\
& $25^{\circ} \mathrm{C}$ & + & ++ & + & ++ & + & + & ++++ & ++++ \\
\hline \multirow{2}{*}{4} & $13^{\circ} \mathrm{C}$ & ++++ & + & +++ & +++ & ++ & +++ & ++ & ++++ \\
& $25^{\circ} \mathrm{C}$ & ++ & ++ & +++ & ++ & ++ & + & +++++ & ++++ \\
\hline \multirow{2}{*}{4.7} & $13^{\circ} \mathrm{C}$ & ++ & + & + & +++ & + & + & ++++ & ++ \\
& $25^{\circ} \mathrm{C}$ & +++ & + & ++ & ++ & + & + & ++++ & + \\
\hline
\end{tabular}

\subsection{The mnn1 Mutation in Combination with Other mnn Improves Killer Resistance}

The mnn 1 mutant is defective in $\alpha 1-3$ mannose addition. The lack of such mannoses results in a more exposed negative charge (due to phosphate groups) than wild type. On the contrary, the mnn6 mutant is defective in the phosphate addition to N-linked chains, which results in a drastic reduction in negative charge to $<5 \%$ of the wild type. It seems that the more exposed negative charge in $\mathrm{mnn} 1$ results in an increment of killer resistance, when compared to wild type.

In some cases, the data showed a level of improvement regarding to the single mutation when it is combined with mnn 1 (higher negative charge) and no change in sensitivity with mnn6. In Table 2, the kbarr1 killer effect on representative mutants is shown.

Table 2. Killer kbarr1 as representative data. Other data not shown affirm that a simple mutation with $m n n 1$ with a proportionally higher negative charge improves the toxin resistance, whereas in combination with mnn6, it is more or equally sensitive.

\begin{tabular}{cccccc}
\hline $\mathrm{pH} / \mathrm{T}$ & mnn1 & mnn6 & mnn10 & mnn1mnn10 & mnn6mnn10 \\
\hline $3.5 / 13^{\circ} \mathrm{C}$ & + & +++ & ++++ & + & ++++ \\
$4 / 13^{\circ} \mathrm{C}$ & + & ++ & ++++ & ++ & ++++ \\
$4.7 / 13^{\circ} \mathrm{C}$ & + & + & ++ & ++ & +++ \\
\hline
\end{tabular}

\subsection{Kbarr1 Is a Good Killer Candidate for Co-Inocula}

Kbarr1 killer toxin, present in Toluraspora delbruekii (EX1180 in this work) has been widely studied by our research group [6]. The mechanism seems to be similar to K1 and $\mathrm{K} 2$ by interacting with beta-glucan in the cell wall. It makes sense that a yeast with reduced size of the N-linked oligosaccharides of mannoprotein could be more sensitive because the toxin reaches the receptor easily. 
Table 3 show the results of the killer behavior of several killer strains on several single mutants. Kbarr1 and K2 have similar killer efficiency. EX1180 (kbarr1) is a Torulaspora delbruekii which has proved to be very interesting concerning the final characteristics in wine.

Table 3. Killer effect of different killer strains on single $m n n$ mutants at $13^{\circ} \mathrm{C}$ and $\mathrm{pH} 3.5$.

\begin{tabular}{cccccccccc}
\hline Killer & Strains & wt & mnn1 & mnn2 & mnn3 & mnn5 & mnn6 & mnn9 & mnn10 \\
\hline K1 & F166 & ++ & + & + & + & + & + & +++ & +++ \\
K2 & E7AR1 & ++ & ++ & ++ & + & + & ++ & ++++ & +++ \\
K28 & F182 & - & - & - & - & - & - & - & - \\
klus & EX229 & - & + & + & + & + & + & + & - \\
kbarr1 & EX1180 & ++ & + & ++ & +++ & ++ & ++ & +++++ & ++++ \\
kbarr2 & EX1257 & - & - & - & - & - & - & - & - \\
no killer & 2K- & - & - & - & - & - & - & - & - \\
\hline
\end{tabular}

Table 4 shows the results of a similar experiment including double mutants.

Table 4. Killer effect of different killers in double mutants.

\begin{tabular}{ccccccccc}
\hline Killer & Strains & mnn1mnn6 & mnn1mnn2 & mnn2mnn6 & mnn6mnn10 & mnn1mnn10 & mnn1mnn9 & mnn2mnn10 \\
\hline K1 & F166 & ++ & + & + & +++ & ++ & +++ & ++ \\
k2 & E7AR1 & ++ & ++ & ++ & +++ & - & +++ & +++ \\
K28 & F182 & - & - & - & - & - & - & - \\
klus & EX229 & + & + & + & ++ & + & + & + \\
kbarr1 & EX1180 & ++ & + & +++ & ++++ & + & +++ & + \\
kbarr2 & EX1257 & - & - & - & - & - & - & - \\
no killer & 2K- & - & - & - & - & - & - & - \\
\hline
\end{tabular}

\section{Conclusions}

(a) Once the relation of the size charge of mannoprotein and effects is clarified, it will be possible to choose mannoproteins on demand (size charge) in order to improve some characteristics of wine, like reducing tartaric or protein precipitation, or improving clarification, mouthfeel, aroma, etc.

(b) The use of co-inocula with the mutants which synthesize the desired mannoprotein and the appropriate killer strain will allow the direct release of mannoprotein from the mutant, avoiding the purification step.

(c) Torulaspora delbruekii EX1180 (Kbarr1) has proved to be a good candidate to be used as the killer counterpart of the co-inoculum.

Author Contributions: Conceptualization, funding acquisition, writing and editing, project administration, L.M.H. Writing original draft, investigation, P.G. Methodology and resources, P.G., A.M. Supervision: J.B., R.V., M.R. All authors have read and agreed to the published version of the manuscript.

Funding: Projects GR18117 and IB16132 (Consejería de Economía, Ciencia y Agenda Digital, Junta de Extremadura), project AGL2017-87635-R (Ministerio de Economía, Industria y Competitividad, Agencia Estatal de Investigación, Gobierno de España) and Fondo Europeo de Desarrollo Regional (FEDER).

Institutional Review Board Statement: Not applicable.

Informed Consent Statement: Not applicable.

Data Availability Statement: Not applicable.

Acknowledgments: Patricia Gil is a recipient of a pre-doctoral fellowship from Junta de Extremadura.

Conflicts of Interest: The authors declare no conflict of interest. 


\section{References}

1. Alexandre, H.; Guilloux-Benatier, M. Yeast autolysis in sparkling wine-A review. Aust. J. Grape Wine Res. 2006, 12, $119-127$.

2. Cebollero, E.; Rejas, M.T.; Gonzalez, R. Autophagy in wine making. In Autophagy: Lower Eukaryotes and Non-Mammalian Systems, Ptar A; Elsevier Academic Press Inc.: San Diego, CA, USA, 2008; Volume 451, pp. 163-175.

3. Ballou, C.E. Isolation, characterization and properties of Saccharomyces cerevisiae mnn mutants with nonconditional protein glycosylation defects. Methods Enzymol. 1990, 185, 440-470.

4. Wickner, R.B. Double-stranded RNA viruses of Saccharomyces cerevisiae. Microbiol. Rev. 1996, 60, $250-265$.

5. Maqueda, M.; Zamora, E.; Alvarez, M.L.; Ramirez, M. Characterization, ecological distribution, and population dynamics of Saccharomyces sensu stricto killer yeasts in the spontaneous grape must fermentations of Southwestern Spain. Appl. Environ. Microbiol. 2012, 78, 735-743.

6. Ramirez, M.; Velazquez, R.; Maquedal, M.; Lopez-Pineiro, A.; Ribas, J.C. A new wine Torulaspora delbrueckii killer strain with broad antifungal activity and its toxin-encoding double-stranded RNA virus. Front. Microbiol. 2015, 6, 983. 